\title{
Reduction of Spin Glasses applied to the Migdal-Kadanoff Hierarchical Lattice
}

\author{
Stefan Boettcher ${ }^{1, *}$ \\ ${ }^{1}$ Physics Department, Emory University, Atlanta, Georgia 30322, USA
}

(Dated: October 21, 2018)

\begin{abstract}
A reduction procedure to obtain ground states of spin glasses on sparse graphs is developed and tested on the hierarchical lattice associated with the Migdal-Kadanoff approximation for lowdimensional lattices. While more generally applicable, these rules here lead to a complete reduction of the lattice. The stiffness exponent governing the scaling of the defect energy $\Delta E$ with system size $L, \sigma(\Delta E) \sim L^{y}$, is obtained as $y_{3}=0.25546(3)$ by reducing the equivalent of lattices up to $L=2^{100}$ in $d=3$, and as $y_{4}=0.76382(4)$ for up to $L=2^{35}$ in $d=4$. The reduction rules allow the exact determination of the ground state energy, entropy, and also provide an approximation to the overlap distribution. With these methods, some well-know and some new features of diluted hierarchical lattices are calculated.

PACS number(s): 05.50.+q, 75.10.Nr, 02.60.Pn.
\end{abstract}

\section{INTRODUCTION}

We propose a set of reduction rules applicable to spin glasses at $T=0$ on any sparse graph and arbitrary bond distribution. These reductions strip graphs of all variables that are connected to at most two neighbors while accounting exactly for the ground state energy, entropy, and approximately for the overlap distribution of the system. In this paper, we introduce these reduction rules, and test them on the hierarchical lattice (see Fig. 1) obtained from the Migdal-Kadanoff bond-moving scheme 1]. The recursive nature of the hierarchical lattice permits us to quickly reduce the equivalent of $10^{9}$ graphs with lengths corresponding to $L=2^{100}$ in $d=3$ and $L=2^{35}$ in $d=4$ dimensions, limited only by accumulating rounding errors. Throughout this paper we focus exclusively on $d=3$ and 4 , and use only a discrete bond distribution,

$$
P(J)=p \delta\left(J^{2}-1\right)+(1-p) \delta(J) .
$$

But our procedure is equally applicable to any continuous bond distribution, such as a Gaussian.

The reduction produces high-accuracy results for the scaling of the defect energy width $\sigma(\Delta E)$ with $L$. We also study the diluted lattice, where $p$ shall refer to the fraction of occupied bonds, and show that this scaling emerges for all $p>p^{*}$. The critical point $p^{*}=0.31032[2]$ is particular to the bond distribution in Eq. (11) and located just above the percolation point $p_{c}=0.281837$ of the lattice. Since $p_{c}$ is a purely topological property of the lattice itself, having $p>p_{c}$ is merely a necessary condition for long-range correlated behavior in a spin glass. Cooperative effects from the bond disorder suppress correlations even for $p_{c}<p<p^{*}$, as was already discussed in Ref. 2]. Correspondingly, the moment $\langle|q|\rangle$ of the overlap distribution [3] becomes non-zero only at $p^{*}$, while the ground state energy and entropy remain smooth for

*Electronic address: www.physics.emory.edu/faculty/boettcher

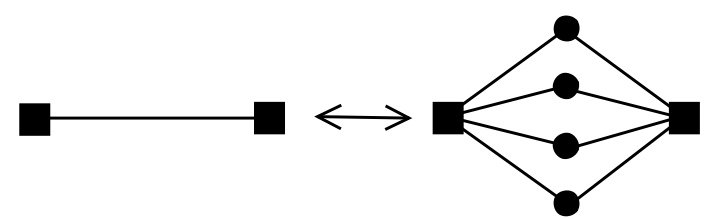

FIG. 1: Recursive generation of the hierarchical lattice, proceeding from left to right. The reduction rules proceed from right to left, replacing the sub-graph with a reduced bond.

all $0 \leq p \leq 1$, even at the transition. Both, entropy and overlap, exhibit an extremum at some $p>p^{*}$ which can be explained in terms of the peculiar lattice hierarchy.

This study suggests that the reduction method provides a powerful means to explore the (potential) onset of replica symmetry breaking (RSB) [3] on finitedimensional spin glasses for arbitrary bond distributions at $T=0$ but variable $p$. The onset of glassy behavior (albeit not RSB) in the Migdal-Kadanoff model at $p^{*}$, close to and intrinsically linked with $p_{c}$, may suggest such a close link between percolation and the possible onset of RSB in realistic lattice models, which we are currently exploring [4]. In fact, we already found a transition for the stiffness exponent in real three- and four-dimensional Edwards-Anderson models, resembling the one observed here, at a $p^{*}>p_{c}[5]$.

Considered in terms of the average connectivity $\alpha=$ $2 d p \sim O(1)$ of spins, dilute lattice glasses as well as the hierarchical lattice at $T=0$ behave strikingly similar to many mean-field models, which are currently studied in the context of combinatorial optimization [6, 7]. Those models also enter into a glassy state (exhibiting RSB) at some finite connectivity $\alpha^{*}$, above a finite-connectivity percolation transition $\alpha_{c}<\alpha^{*}$. But in mean-field models the onset of RSB is often studied as a precursor to yet another critical point, the satisfiability transition [9], whereas geometrically defined models are unsatisfiable at any finite connectivity. These features should be independent of the bond distribution, assuming zero mean 
and unit variance.

While the hierarchical lattice is completely reducible, diluted realistic lattices will become irreducible soon above percolation, in which case our algorithm [5] is used to reduce any graph as much as possible first, followed by a complete exploration of the ground states of the far more compact remainder graph using the extremal optimization heuristic [10]. With this hybrid approach, we are typically able to compute ground state energies, entropies and overlaps for graphs 10-100 times larger than previously recorded, extending well beyond the percolation point.

\section{MIGDAL-KADANOFF HIERARCHICAL LATTICE}

To illustrate the reduction method, and to test our algorithm, we consider here the hierarchical lattice (see Fig. 1), obtained in the Migdal-Kadanoff realspace renormalization scheme 1] for low-dimensional spin glasses. These lattices have a simple recursive, yet geometric, structure and are well-studied 2, 8, 11, 12, 13, 14]. Most importantly, they are completely reducible, and we can discuss the method independently of any subsequent optimization that may be required for more complicated models. The most interesting property of these lattices is the curious fact that the scaling of its defect energy distribution behaves very similar to that measured for actual two- and three-dimensional lattices [5, 8, 15].

As described in Fig. 11 starting from generation $I=0$ with a single link, at each subsequent generation $I+1$, all links from $I$ are replace with a new subgraph. The structure of the subgraph arises from the bond-moving scheme in $d$ dimensions, and has $2^{d}=8$ links for $d=3$ here. Thus, a hierarchical lattice of generation $I$ has $l_{I}=\left(2^{d}\right)^{I}$ links, thus corresponding to a $d$-dimensional lattice of "length" $L=2^{I}$ but $n_{I}=2+2^{d-1}\left(L^{d}-1\right) /\left(2^{d}-\right.$ $1)=O\left(L^{d}\right)$ vertices. While the average connectivity is $2 l_{I} / n_{I} \sim 4-2^{2-d}$, the two root-vertices from generation $I=0$ themselves obtain in generation $I \gg 1$ a connectivity of $\sim 2^{(d-1) I}$, and $\sim 2^{d I-1}$ vertices are only two-connected, i. e. 7 in 8 for $d=3$.

The diluted hierarchical lattice percolates when there is a path between the two root-vertices. This notion leads to a simple recursion relation for the percolation threshold by counting the weights of all diluted subgraphs from Fig. [1 that percolate:

$$
p_{I+1}=4 p_{I}^{2}-6 p_{I}^{4}+4 p_{I}^{6}-p_{I}^{8},
$$

which has a non-trivial stationary point at $p_{c}=$ 0.2818376366. It has been pointed out by Ref. [2] that a spin glass with the discrete $\pm J$-bond distribution in Eq. (11) exhibits instead a critical transition between a paramagnetic and a spin glass phase at $p^{*}=0.31032$, which is closely related to the percolation transition. While below $p_{c}$ disconnected bonds $(J=0)$ clearly dominate and prevent long-range correlations, even for $p_{c}<p<p^{*}$ such correlations remain suppressed due to the cooperative behavior of parallel bond structure pervasive in the lattice that lead to many cancellations (see "double rule" in Sec. IIII) and additionally disconnects subgraphs at some higher level of the hierarchy.

\section{REDUCTION RULES FOR SPIN GLASSES}

In the Ising spin glass problem we assign to each vertex $i$ of a graph or lattice, $1 \leq i \leq n$, a spin variable $x_{i} \in\{-1,+1\}$, while each link between two connected vertices $i$ and $j$ obtains a bond variable $J_{i, j}$ that is drawn at random from some distribution $P(J)$, which may be discrete or continuous. The problem consists of finding spin configurations $\left(x_{1}, \ldots, x_{n}\right)$ which minimize the Hamiltonian, or total energy,

$$
H\left(x_{1} \ldots, x_{n}\right)=-\sum_{<i, j>} J_{i, j} x_{i} x_{j}
$$

for a fixed (quenched) set of bonds $J_{i, j}$. Of course, for $J_{i, j}>0$ (only ferromagnetic bonds), the solution is simply to make all connected spins aligned with each other. But if some or all bonds are negative, and spins are sufficiently connected, the problem can be frustrated [16] and solutions become nontrivial.

In the following, we explain the rules by which to eliminate recursively all two-connected spins while accounting for the energy, entropy, and approximate overlap of ground-state configurations. To this end we have to generalize bonds by adding internal degrees of freedom, which evolve during the reduction process. Each bond between two spins $i$ and $j$ now consists of a tuple

$$
\mathcal{J}_{i, j}=\left(J_{i, j}, m_{i, j}^{+}, m_{i, j}^{-}, s_{i, j}^{+}, s_{i, j}^{-}\right),
$$

where $J_{i, j}$ is the weight of the bond, $m_{i, j}^{ \pm}$is the entropy accumulated by the bond, and $s_{i, j}^{ \pm}$counts the number of previously reduced spins whose state is completely determined ("entrained") by the bond for $x_{i} x_{j}= \pm 1$, respectively. In addition, there is an energy offset $E_{\text {offset }} \leq 0$ accounting for the energy difference between the original and the reduced graph. In general, determining entropy and overlap from the $m$ - and $s$-values can be quite complicated for the interesting case of an irreducible remainder graph [4]. For the completely reducible hierarchical lattice, it is a lot simpler and we will describe it below. Initially, for the unreduced graph, all $m$ - and $s$-values and $E_{\text {offset }}$ are zero, and the bonds are drawn from one of the usual distributions, for example, Eq. (11).

These rules are elaborate but easily enumerated, independent of the previous or future structure of the graph or the spin configuration. A more complete set of rules including zero-, one-, and three-connected vertices required for arbitrary graphs will be presented elsewhere [4, 5]. There, each spin $i$ obtains one additional number $s_{i}$ (aside from its state $x_{i}$ ) that counts the previously eliminated spins whose state is entrained to $i$. 
To demonstrate the use of these extra degrees of freedom, consider, for instance, the reduction of a oneconnected spin $j$ : It and its bond $\mathcal{J}_{i, j}$ to its sole neighbor $i$ disappears from the graph, and the connectivity of $i$ is decremented. The new graph will have itself a ground state energy offset by $\left|J_{i, j}\right|$ relative to the unreduced graph, i. e. $E_{\text {offset }} \leftarrow E_{\text {offset }}-\left|J_{i, j}\right|$. For $J_{i, j}=0$ (a possibility we eventually have to allow for, even if our unreduced bond distribution did not), all information contained in $\mathcal{J}_{i, j}$ is discarded and $s_{i}$ doesn't change, since $x_{j}$ is free to take on any value \pm 1 in the ground state; only the entropy of the old graph is offset by $\ln 2$ with respect to the new graph. In turn, for $J_{i, j}>0\left(J_{i, j}<0\right)$, $j$ is entrained to the state of $i$ in any ground state configuration, and $s_{i}$ is incremented. If the bond $\mathcal{J}_{i, j}$ had previously inherited nonzero entropies $m_{i, j}^{ \pm}$and entrainments $s_{i, j}^{ \pm}$, there would be an offset in entropy by $m_{i, j}^{+}$ $\left(m_{i, j}^{-}\right)$between the reduced and the original graph, and $s_{i}$ would be further increased by $s_{i, j}^{+}\left(s_{i, j}^{-}\right) ; m_{i, j}^{-}\left(m_{i, j}^{+}\right)$and $s_{i, j}^{-}\left(s_{i, j}^{+}\right)$are discarded because the ground state must have $x_{i} x_{j}=+1\left(x_{i} x_{j}=-1\right)$.

With the two-point rule we use exclusively in this paper, a spin variable $x_{i}$ and its two bonds $\mathcal{J}_{1}$ and $\mathcal{J}_{2}$ are replaced by a new bond $\mathcal{J}$. It is easy to show that

$$
J=\frac{1}{2}\left(\left|J_{1}+J_{2}\right|-\left|J_{1}-J_{2}\right|\right),
$$

which assigns to $J_{j, k}$ the bond with the smaller modulus and the sign of $J_{1} J_{2}$. The energy offset $E_{\text {offset }}$, accounting for the energy of the original graph, will have to be lowered by

$$
\frac{1}{2}\left(\left|J_{1}+J_{2}\right|+\left|J_{1}-J_{2}\right|\right) .
$$

At this level, our reduction rules correspond to the familiar traces (at $T=0$ ) used many times before to study the hierarchical lattice (see, e. g., Refs. 13, 15]).

Clearly, for $\left|J_{1}\right| \neq\left|J_{2}\right|$, the reduced spin $i$ is entrained to the neighbor with the bond of larger modulus, while the weaker bond may be violated. The entropy and entrainment variables that $\mathcal{J}$ inherits from the two reduced bonds are controlled by the sign of the stronger one. We can summarize these rules by defining $\mathcal{J}_{>}$and $\mathcal{J}_{<}$as the bond with the stronger and weaker (in modulus) weight, respectively. Then, we obtain for the new bond:

$$
\begin{aligned}
\frac{\left|J_{>}\right|>\left|J_{<}\right|}{m^{ \pm}} & : \\
s^{ \pm} & =m_{>}^{\operatorname{sign}\left(J_{>}\right)}+m_{<}^{ \pm \operatorname{sign}\left(J_{>}\right)}+s_{<}^{ \pm \operatorname{sign}\left(J_{>}\right)},
\end{aligned}
$$

The case $\left|J_{1}\right|=\left|J_{2}\right|$ leads to further distinctions:

$$
\begin{aligned}
m^{ \pm} & = \begin{cases}m_{1}^{\operatorname{sign}\left(J_{1}\right)}+m_{2}^{\operatorname{sign}\left(J_{2}\right)} & , J_{1}= \pm J_{2} \neq 0 \\
\ln \left(e^{m_{1}^{+}+m_{2}^{ \pm}}+e^{m_{1}^{-}+m_{2}^{\mp}}\right) & , J_{1}=\mp J_{2}\end{cases} \\
s^{ \pm} & = \begin{cases}s_{1}^{\operatorname{sign}\left(J_{1}\right)}+s_{2}^{\operatorname{sign}\left(J_{2}\right)} & , J_{1}= \pm J_{2} \neq 0 \\
0 & , J_{1}=\mp J_{2}\end{cases}
\end{aligned}
$$

Finally, we need a rule to combine double bonds, which will be created eventually. When two bonds, $\mathcal{J}_{1}$ and $\mathcal{J}_{2}$, connect the same two spins, they are simply replaced with a new bond

$$
\mathcal{J}=\mathcal{J}_{1}+\mathcal{J}_{2}
$$

It is important to note that the two-point and double rules only effect the newly created bond and the energy offset, but not the two neighboring spins (or the entropy offset). Unlike for the one-point rule, we do not need to consider an internal entrainment number for spins here.

\section{ALGORITHM AND IMPLEMENTATION}

With the reduction rules from Sec. III it is now straightforward to evaluate hierarchical lattices of any size. In effect, these rules allow us to reverse the generating mechanism depicted in Fig. 10 Recursively, from one generation to the next, we reduce all two-connected spins in the middle of the subgraph first, then use the doublebond rule sequentially, until a single, reduced bond is left. Once an entire graph has been reduced into such a single bond, the weight $J$ of that bond is in fact half the defect energy, $\Delta E=2 J$, of that graph, i. e. the energy difference between having both root spins aligned and anti-aligned. The entropy of that graph is simply given by $m^{\operatorname{sign}(J)}$, or by $\ln \left[\exp \left(m^{+}\right)+\exp \left(m^{-}\right)\right]$for $J=0$. The energy of the graph can be obtained from the running energy-offset $E_{\text {offset }}$, accounting for the reduction of bonds along the way, and $J$. For our purposes, it is more useful to extract the (non-negative) "cost" of a graph given by the absolute weight of all violated bonds for any bond distribution. In case of the discrete $\pm J$ distribution in Eq. (1), this sum reduces to a count of all violate bonds (all of absolute weight 1$)\left(l+E_{\text {offset }}-|J|\right) / 2$, where $\langle l\rangle=p L^{d}$ is the average number of non-vanishing bonds in a graph. As an approximate measure of the overlap, we merely store the largest entrainment number $s_{\max }$ observed at any point during the reduction process, which is a measure of the largest correlated cluster of spins within the lattice. The size of this cluster will either dominate the overlap (with smaller clusters adding some negligible fluctuations) in the spin glass state or be itself a vanishing fluctuation in the paramagnetic state.

Although these measurements are not difficult, the reduction of ever larger graphs soon becomes untenable, since the cost of reducing true graphs would grow by a factor of $2^{d}$ for each generation. Instead, we exploit the fact that each subgraph within the hierarchy is independent of any other parallel subgraph. As above, we reduce all graphs down to a single bond. To assemble a graph of generation $I+1$ we only need a large enough pool of independent graphs of generation $I$. In fact, many nearly-independent graphs of generation $I+1$ can be assembled by repeatedly drawing at random on $2^{d}$ graphs of generation $I$ from that pool. 
We proceed as follows: Assume that we already possess a pool of $A_{I}$ reduced graphs at every generation $I$ (up to a maximal generation $I_{\max }$ ), each graph represented by its bond $\mathcal{J}, E_{\text {offset }}$, and $s_{\max }$. Each cycle of the algorithm generates an elementary subgraph (generation $I=1$ ) with bonds $J$ drawn from the bond distribution in Eq. (1) and empty entropy, entrainment, $E_{\text {offset }}$, and $s_{\max }$ variables. The elementary subgraph is reduced to obtain a bond $\mathcal{J}$ of the first generation, replacing the oldest entry in pool $A_{1}$. After every $k$ th new reduced bond entering into pool $A_{I-1}$, we randomly assemble $2^{d}$ such bonds into a new subgraph to create a new reduced bond to be entered into pool $A_{I}$, as long as $I \leq I_{\max }$.

If we choose $A_{I}=k=2^{d}$ and assemble bonds sequentially instead of at random, this algorithm produces exact hierarchical lattices, where each subgraph is certain to be independent. But to obtain just one graph of, say, generation $I=10$, we have to assemble $2^{30}$ elementary subgraphs for $d=3$. Instead, for all $p$ not too close to $p^{*}$, we simply choose large pools, $A_{I}=2048$, and as small as $k=1$. That is, each time we assemble an elementary graphs at $I=0$, we enter its value to pool $A_{1}$, assemble a subgraph out of $A_{1}$, add the reduced bond to pool $A_{2}$, etc, all the way up to $I_{\max }$ in each cycle. Hence, our implementation becomes independent of $L$, and for instance at $p=1$ we have assembled graphs up to generation $I_{\max }=100$ in $d=3$ and $I_{\max }=35$ in $d=4$, corresponding to $L=2^{100}$ or $2^{3} 5$, respectively. $I_{\max }$ is only limited by apparent limits on floating point accuracy due to repeated cancellation between almost equal bonds. Note that each pool gets completely refreshed after $A_{I}$ creations of new graphs from the pool just below it, while there are about $O\left(2^{d A_{I}}\right)$ different subgraphs that could be created out of each pool at any one time.

Every time a graph gets added to a pool, its properties are evaluated from its $\mathcal{J}, E_{\text {offset }}$, and $s_{\max }$. While ground state energy, entropy, and (approximate) overlap converge quickly with $I$, and throughout are quite independent of the choice of $k$ and $A_{I}$, the measurement of the defect energy becomes quite sensitive to the algorithmic parameters near $p^{*}$. In those cases, we increase $k$ (and lower $I_{\max }$ correspondingly), until stable results were obtained. Only at $p \approx p^{*}$, the exact algorithm was necessary to observe the true defect energy distribution.

\section{NUMERICAL RESULTS}

Using the algorithm from Sec.IV we have studied various properties of a Ising spin glass with bond distribution as given in Eq. (11) on the diluted and undiluted hierarchical lattice. In fact, using the easiest implementation with $k=1$, pool sizes of $A_{I}=2048$, and $I_{\max }=20$, we first scanned lattices for $0<p \leq 1$ in steps of $\Delta p=0.1$. At each $p$ and $I$, we averaged over $10^{7}$ values. We found that for each $p$, the ground state energies per spin, entropies per spin, and the moment of the overlap, $\langle|q|\rangle$ were well-converged at $I_{\max }=20$, and insensitive to the

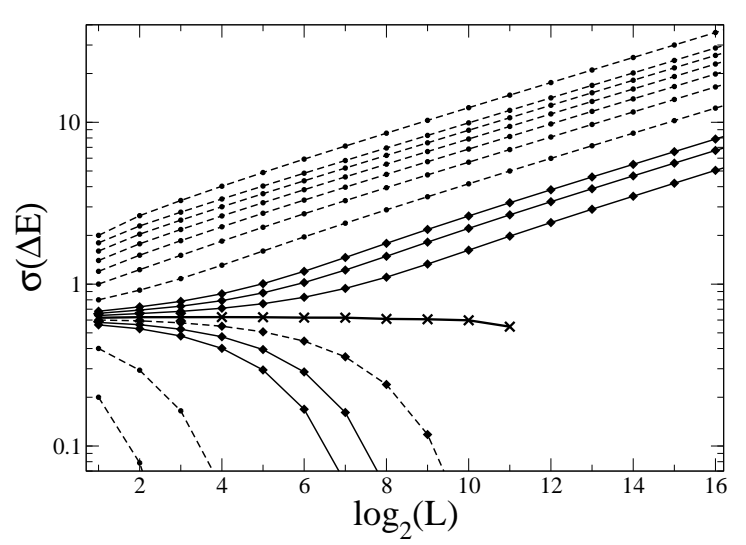

FIG. 2: Plot of the width $\sigma(\Delta E)$ of the defect energy distribution as a function of systems size $L$ in $d=3$ for various bond fractions $p$. Dashed lines from bottom to top refer to $p=0.1,0.2, \ldots, 1$, while data with diamonds and crosses refer to $p=0.28,0.29, \ldots, 0.34$. The data points with circles were obtained using the $k=1$ implementation from Sec. IV] those with diamonds using $k=2$, and those with crosses at $p=0.31 \approx p^{*}$ required the exact algorithm. Note that for $p<p^{*}$ the width evolves toward the $p=0$ fix-point with $\sigma(\Delta E)=0$, while for $p>p^{*}$ it invariably evolves to the $p=1$ fix-point with scaling behavior $\sigma(\Delta E) \sim L^{y}$. While the exact asymptotic behavior emerges only for very large sizes $L$ when $p \approx p^{*}$, it is already obvious for small $L$ whether $\sigma(\Delta E)$ is going to rise or to fall.

choice of $k$ using trials with $k=2$.

We also found the width of the defect energy distribution, $\sigma(\Delta E)=\sqrt{\left\langle\Delta E^{2}\right\rangle-\langle\Delta E\rangle^{2}}$, to be stable except for $p=0.3$. For large $I=\log _{2}(L)$ the distribution of defect energies either collapses into a $\delta$-function at zero for small $p$, or eventually converges to the distribution of the undiluted lattice $(p=1)$. Near $p \approx p^{*}$, the evolution of that distribution becomes highly sensitive to small fluctuations, and the minute statistical dependencies inherent in our algorithm for small $k$ escalate. Thus, we ran another scan for $0.28 \leq p \leq 0.34$ using $k=2$ and $10^{4}$ graphs at $I_{\max }=20$ (and hence gaining a factor $k$ more graphs at each lower generation $I$ ). Now, only for $p=0.31 \approx p^{*} \operatorname{did} \sigma(\Delta E)$ become unstable (by comparison with trial runs at $k=4)$. Finally, for $p=0.31$, we used an exact algorithm and indeed found $\sigma(\Delta E)$ to be horizontal for many generations until it eventually decays, indicating that $p^{*} \gtrsim 0.31$, consistent with the expected value of 0.31032 from Ref. 2]. The results for $\sigma(\Delta E)$ as a function of $I=\log _{2}(L)$ for all $p$ are shown in Fig. 2]

In Figs. 35 we plot the number of violated bonds (or cost) per spin, entropy per spin, and a characteristic moment of the (approximate) overlap distribution for $L \rightarrow \infty$ and $d=3$. The cost is already non-zero for small $p$, rising consistent with $p^{4}$, proportional to the probability of creating a small, frustrated loop that requires four bonds. Just beyond $p^{*}$, the cost per spin rises more slowly, but without drastic changes for any $p$. Similarly, 


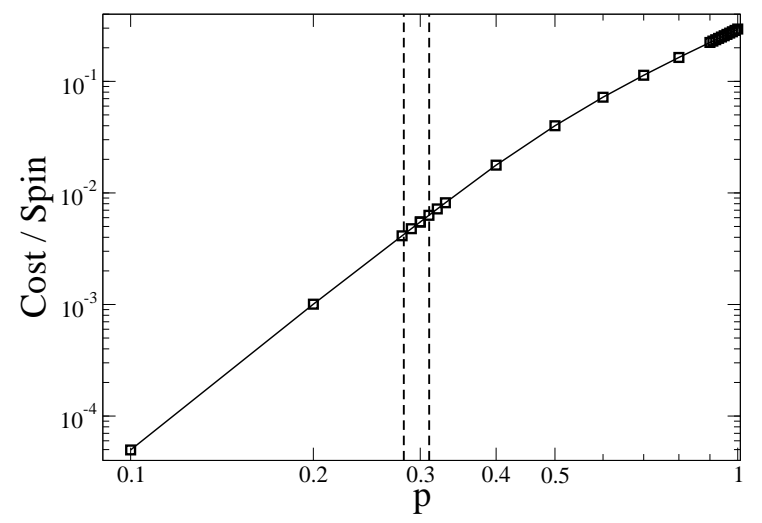

FIG. 3: Log-log Plot of the cost per spin for the infinite system in $d=3$ as a function of $p$. This cost, i. e. the fraction of violated bonds, only approaches zero for $p \rightarrow 0$ (consistent with $p^{4}$ ). The data smoothly continues through both critical points at $p_{c}$ and $p^{*}$, indicated by dashed vertical lines. For $p>p^{*}$ the cost appears to rise less rapidly than for $p<p^{*}$.

the entropy density in Fig. 4 remains unaffected by the transition at $p^{*}$, as is expected [17]. Instead, it reaches a minimum at about $p=0.7$ that is an artifact of the hierarchical lattice. The large majority of spins in the graph (a fraction $\sim 7 / 8$ for $d=3$ ) are at most two-connected spins whose contribution dominates the entropy density. In a fully bonded graph $(p=1)$, about half of these spins are free, the other half is constraint, with an energy density of $\ln (2) / 2$. For small dilutions $p \lesssim 1$, about every removed bond turns a two-connected spin into a oneconnected spin, which is always constrained, and the entropy declines. Eventually, at large dilution ( $\operatorname{small} p$ ), an increasing number of one-connected spins become totally disconnected and free again, raising to entropy density to its ultimate value, $\ln (2)$, for the unconnected $(p=0)$ lattice. By this argument the entropy density should be given simply by

$$
\frac{S}{n} \approx\left[(1-p)^{2}+\frac{1}{2} p^{2}\right] \ln (2),
$$

which fits the data exceedingly well.

In contrast with energy and entropy, the overlap distribution is clearly sensitive to the transition from paramagnetic to glassy behavior at $p^{*}$, as shown in Fig. [5 There, we plot only its moment, since the distribution itself quickly converges to a structureless $\delta$-peak as expected for a model that is replica symmetric (RS) throughout. In fact, we find that $\langle|q|\rangle / \sqrt{\left\langle q^{2}\right\rangle} \sim 1$ to within $1 \%$ for all $p$. (For one, a broad overlap distribution indicative of widely separated ground state configurations and RSB $[\underline{3}$ would be antithetical to the reducibility of the hierarchical lattice.) About at $p^{*}$, the location of the peak becomes non-zero and splits into two symmetric peaks, leading to a non-zero moment $\langle|q|\rangle$.

The moment experiences a surprising maximum very close to $p=1$, varying sharply for small dilutions. Again, the maximum may be explained in terms of the

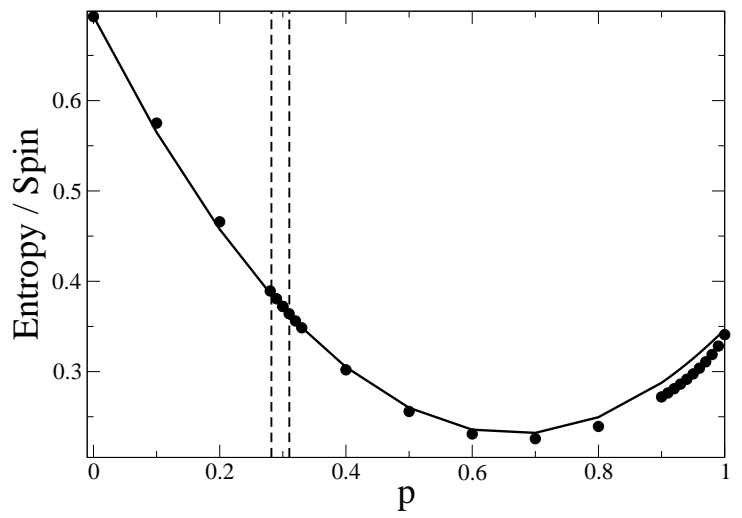

FIG. 4: Plot of the entropy per spin for the infinite system as a function of $p$. The data smoothly continues through both critical points at $p_{c}$ and $p^{*}$, indicated by dashed vertical lines, but exhibits a surprising minimum. The data is approximated well by Eq. (10) (continuous line), as discussed in the text.

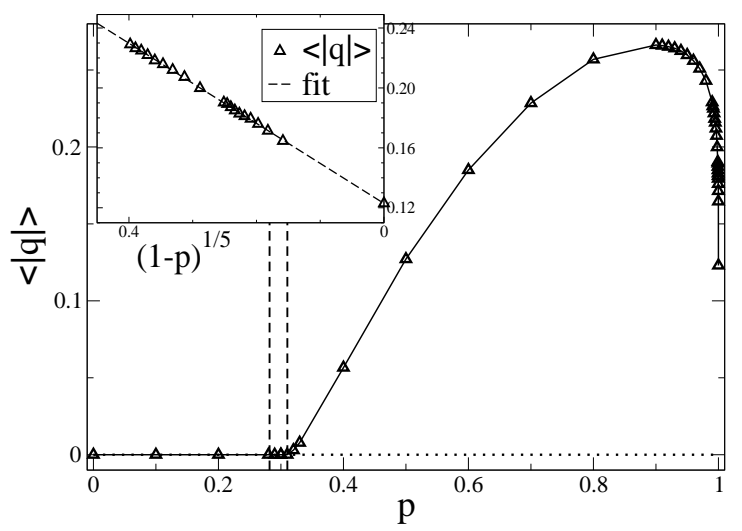

FIG. 5: Plot of the moment $\langle|q|\rangle$ of the overlap distribution as a function of $p$. The moment remains zero for all $p \leq p^{*}$, including at the percolation point $p_{c}$. (Both, $p_{c}$ and $p^{*}$, are marked by dashed vertical lines.) Above the transition at $p^{*}$ to a spin glass state, $\langle|q|\rangle$ rises first linearly, but then turns over at a maximum at small dilutions just below $p=1$, and drops sharply at $p=1$. The inset shows that $\langle|q|\rangle$ seems to scale with $(1-p)^{1 / 5}$ for $p \rightarrow 1$; the dashed line is a linear fit.

abundance of two-connected spins: Whatever the size of strongly correlated spin clusters may be in the fully connected lattice $(p=1)$, the overwhelming conversion of two-connected to one-connected spins at small dilution can only increase the size of those clusters at its edge, since one-connected spins are always entrained. But more importantly, the elimination of two-connected spins, say, within a subgraph as shown in Fig. 1 will enhance the probability that the two root-spins of that subgraph become correlated, which would merge the clusters that those spins are part of. While the latter effect could suddenly increase the size of typical clusters by a factor, it is not clear whether the steep slope observed at $p=1$ is not an artifact of our approximation to the overlap (see Sec. IV]. Further numerical studies suggest that

$$
\langle|q|\rangle_{p}-\langle|q|\rangle_{p=1} \sim(1-p)^{\frac{1}{5}} \quad(p \rightarrow 1),
$$


as seen in the inset of Fig. 15 We have not found an explanation for the scaling in Eq. (11) in terms of the lattice yet, since it is also a property of the entrainment in the spin glass state (and hopefully, the overlap).

As shown in Fig. 2 for all $p>p^{*}$ we obtain the expected scaling of the width of the defect energy distribution with system size $L$,

$$
\sigma(\Delta E) \sim a L^{y}[1+\epsilon(L)],
$$

for large enough $L$, including a next-to-leading correction $\epsilon(L) \ll 1$. Most conveniently, it appears that our $k=1$ algorithm (for large enough pools $A_{I}$ ) described in Sec. IV is least likely to create spurious dependencies for $p=1$, where we would expect the quickest and most stable convergence toward the renormalized bond distribution. Thus, we disabled the sampling of energyoffsets, entropies, and entrainments, which already are well-converged at $I_{\max }=20$, and only focused on the reduction of bonds. Setting $I_{\max }=100(d=3)$ or $I_{\max }=40(d=4), k=1$, and $A_{I}=1024$ for each $I$, we measured more than $10^{9}$ reduced bonds at each $I$ to obtain the distributions of defect energies $\Delta E=2 \mathrm{~J}$ in $d=3$ and 4 . (In fact, we repeated the same calculation with a different random number generator, without markable difference in results. A previous run with $I_{\max }=150$ in $d=3$ showed divergence due to limited floating point precision at $I \approx 120$; the same problem emerged already at $I_{\max }=40$ in $d=4$.) At that level, we obtained a ratio between its absolute mean and its width $\sigma(\Delta E)$ of $<10^{-4}$ in both dimensions, even for the largest $I$, which is a measure of the statistical error (the distribution is supposed to be symmetric).

Surprisingly, although we extended the scaling regime in $d=3$ by a power of 10 compared to previous calculations $\left(L=2^{100}\right.$ compared to $L=2^{10}[13]$ ), the extrapolation for the scaling exponent $y$ in Eq. (12) only yields 5 digits accuracy, see top of Fig. 6] First, we approximated $y \approx 0.2555$ from the intercept of $\log _{2}(\sigma) / \log _{2}(L)$ with respect to $1 / \log _{2}(L)$ at $L \rightarrow \infty$, which displays large corrections to scaling. We are then able to refine our estimate of $y$ by converting Eq. (12) to $\ln (\sigma)-y \ln (L) \sim$ $\ln (a)+\epsilon(L)$, using $\ln (1+\epsilon) \sim \epsilon$. Plotting that expression again vs. $1 / \log _{2}(L)$ (note that $\log _{2}(L)=I$ ) suggest a straight-line asymptotic behavior that would imply $\epsilon \sim b / \log _{2}(L)$ for $L \rightarrow \infty$. The top of Fig. [6]indicates that a value of $y=0.25546(3)$ in $d=3$ leads to an apparently converging result with $\ln (a) \approx 3 / 4$ at the intercept for $L \rightarrow \infty$. We have used the same procedure to find $y=0.76382(4)$ in $d=4$, see bottom of Fig. [6] Although the floating point accuracy here deteriorated already at $L=2^{35}$, the asymptotic scaling regime is reached for much small $L$ than in $d=3$, leading to comparable accuracy in the fit.

Our value for the stiffness exponent $y$ in $d=3$ is $6 \%$ below $y=\left[d-1+\log _{2}(1-2 / \pi)\right] / 2=0.2698$ obtained analytically in Ref. [12] with a Gaussian approximation, and consistent with $y \approx 0.25$ obtained numerically in Ref. [8] by evolving a "pool" of bonds through several genera-
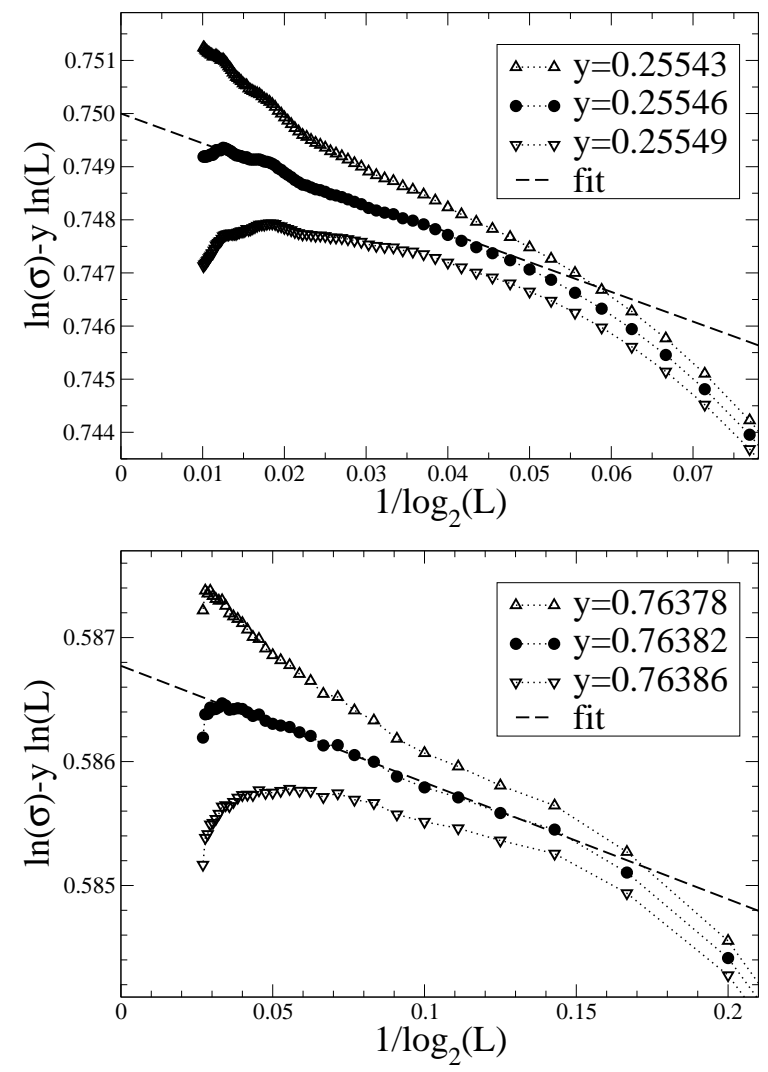

FIG. 6: Extrapolation plot for the leading asymptotic behavior of $\sigma(\Delta E)$ as a function of system size $L$ in $d=3$ (top) and $d=4$ (bottom). The data for $\sigma(\Delta E)$ is converted to $\ln (\sigma)-y \ln (L)$ and plotted vs. the apparent scaling correction, $1 / \log _{2}(L)$, for different estimates of the exponent $y$. For $d=3$ the graph at the center (circles) plots the data for $y=0.25546$ and seems to converge linearly to a finite value of about $3 / 4$ for $L \rightarrow \infty$. In turn, using $y=0.25549$ (down triangle) or $y=0.25543$ (up triangle) on the same data seems to lead tor diverging extrapolations, suggesting $y_{3}=0.25546(3)$. The same procedure for $d=4$ brackets the "best" choice of $y=0.76382$ (circles) between two diverging choices of $y=0.76386$ (down triangle) and $y=0.76378$ (up triangle), suggesting $y_{4}=0.76382(4)$.

tions, very similar to our algorithm in Sec. IV Since both of these references use an initial Gaussian bond distribution, in contrast to our discrete distribution in Eq. (1), the results suggest that the stiffness exponent is independent of the bond distribution. In Ref. [14], an expansion in the limit of large dimension $d \rightarrow \infty$ is used to demonstrate the independence of $y$ of the initial bond distribution. A recursion in the generation $I$ is constructed which for $I \rightarrow \infty$ leads invariably to the Gaussian derived in Ref. 12]. With finite-dimensional corrections, this expansion improves on the Gaussian result above to yield $y_{3} \approx 0.255$, in excellent agreement with our result here. In contrast, we expect that energy, entropy, overlap, and even $p^{*}$ would depend on the distribution at least quantitatively. 


\section{CONCLUSIONS}

We have presented a new method to reduce spin glasses on arbitrary graphical structures. The reductions are most appropriate for sparse graphs near their percolation threshold, which may be either completely (as in this paper) or substantially reduced, facilitating further optimization using standard methods [4, 5]. In either case, the reductions allow to extract exact ground state energies and entropies, and even provide a good approximation to collective properties such as the overlap distribution.

With the reduction method we have reproduced a number of ground-state properties of the Migdal-Kadanoff hierarchical lattice at any dilution, and discovered various new features, which can be mostly explained in terms of the lattice structure. The simplicity of this model further permitted us to determine the exponent $y$ in $d=3$ and 4 for the growth of the defect energy with system size to unprecedented accuracy. We have recently applied the reduction method to diluted Edwards-Anderson models for $d=3$ and 4 , obtaining $y_{3}=0.240(5)$ and $y_{4}=0.60(1)$ for the respective stiffness exponents [5]. These results demonstrate that the Migdal-Kadanoff approximation in $d=3$ is even closer to the Edwards-Anderson value than had been previously expected [8, 15], although they are definitely distinct. The differing values in $d=4$ reflect the fact that Migdal-Kadanoff is a low-dimensional approximation.

The results obtained here and in Ref. [5] suggest that the reduction method may be a useful tool in the exploration of Ising spin glass systems on any graph or lattice near percolation that may reveal the onset of non-trivial configurations of ground states.

\section{Acknowledgments}

I would like to thank A. Percus and M. Paczuski for helpful discussions.
[1] A. A. Migdal, J. Exp. Theo. Phys. 42, 743-746 (1976), and L. P. Kadanoff, Ann. Phys. 100, 359-394 (1976).

[2] A. J. Bray and S. Feng, Phys. Rev. B 36, 8456-8460 (1987).

[3] M. Mezard, G. Parisi, and M. A. Virasoro, Spin Glass Theory and Beyond (World Scientific, Singapore, 1987).

[4] S. Boettcher and A. G. Percus, in preparation.

[5] S. Boettcher, Determining Stiffness Exponents from the Reduction of Dilute Lattice Spin Glasses, cond-mat/0303431

[6] R. Monasson, R. Zecchina, S. Kirkpatrick, B. Selman, and L. Troyansky, Nature 400, 133-137 (1999).

[7] M. Leone, F. Ricci-Tersenghi, and R. Zecchina, J. Phys. A: Math. Gen. 34, 4615 (2001).

[8] A. J. Bray and M. A. Moore, J. Phys. C: Solid State Phys. 17, L463-L468 (1984).

[9] See Frontiers in problem solving: Phase transitions and complexity, eds. T. Hogg, B. A. Huberman, and C.
Williams, special issue of Artificial Intelligence 81:1-2 (1996).

[10] S. Boettcher and A. G. Percus, Phys. Rev. Lett. 86, 52115214 (2001).

[11] S. Kirkpatrick, Phys. Rev. B 15, 1533-1538 (1977).

[12] B. W. Southern and A. P. Young, J. Phys. C: Solid State Phys. 10, 2179-2195 (1977).

[13] B. Drossel and M. A. Moore, Eur. Phys. J. B 21, 589-594 (2001).

[14] J.-P. Bouchaud, F. Krzakala, and O.C. Martin, Energy exponents and corrections to scaling in Ising spin glasses, cond-mat/0212070

[15] K. H. Fischer and J. A. Hertz, Spin Glasses (Cambridge University Press, Cambridge, 1991).

[16] G. Toulouse, Comm. Phys. 2, 115 (1977).

[17] R. Monasson and R. Zecchina, Phys. Rev. Lett 76, 38813885 (1996). 